\title{
Monetización en publicidad digital: interactividad para HbbTV
}

\author{
Joan Francesc FondeVILa Gascón \\ Universitat Ramon Llull, et al. \\ joanfrancesc.fondevila@upf.edu \\ https://orcid.org/0000-0002-6587-939X
}

JORDI BOTEY LÓPEZ

Universitat Ramon Llull

jordibl@blanquerna.url.edu

https://orcid.org/0000-0001-6326-9785

\author{
Josep Rom Rodríguez \\ Universitat Ramon Llull \\ joseprr@blanquerna.url.edu \\ https://orcid.org/0000-0002-4910-2169
}

\author{
FÁtIMA VILA \\ EAE Business School \\ fvila.marquez@campus.eae.es. \\ https://orcid.org/0000-0002-1281-4314
}

\section{Monetization in digital advertising: interactivity for HbbTV}

\begin{abstract}
RESUMEN
El HbbTV promueve la interactividad. En esta investigación analizamos los resultados de una serie sincrónica de campañas de publicidad interactiva llevada a cabo en 2016 por parte de Henkel en un canal de televisión en España. Las campañas eran cuatro propuestas diferenciadas en los paneles de medida de CTR. Se suministró a la cadena de televisión una muestra con cuatro anuncios de un producto de belleza de Henkel. Se observó que el telespectador tiende a clicar el botón de aviso legal (el botón verde), y que existe una relación entre el tiempo promedio de reproducción del vídeo en segundos y la voluntad

de querer participar y un equilibrio entre las diferentes campañas a la hora de impulsar el clic del telespectador.
\end{abstract}

PALABRAS CLAVE

HbbTV, Publicidad interactiva, Televisión interactiva, Comunicación, Marketing, Métricas

\section{ABSTRACT}

HbbTV promotes interactivity. In this study we analyzed the results of a synchronous series of interactive advertising campaigns conducted in 2016 by Henkel on a television channel in Spain. The campaigns were four different proposals in panels measuring CTR. It was supplied to the television a sample with four ads of a beauty product of Henkel. It was observed that the viewer tends to click on the legal warning button (the green button), and that there is a relationship between the average video playback time in seconds and the willingness to participate and a balance between the different campaigns at the time to push the viewer's click.

\section{KEYWORDS}

HbbTV, Interactive advertising, Interactive television, Communication, Marketing, Metrics 


\section{Introducción}

El HbbTV (Hybrid Broadcast Broadband TV) progresa en España al ritmo del factor de convergencia (Reding, 2007; 2008; Del Ama-Gonzalo, Barceló-Ugarte y Sánchez-Martínez, 2011; Vázquez, 2013; Fondevila-Gascón, 2016), obteniendo rédito del apogeo de los medios y las redes sociales (Abertis Telecom, 2012; HighBeam Research, 2018).

El HbbTV inspira el desarrollo de la publicidad interactiva (Azcoitia, 2006), apoyada por el flujo digital congénito a la Sociedad de la Banda Ancha (Fondevila-Gascón, 2012b). La digitalización ha modificado de raíz la realidad de la televisión y del periodismo (Fondevila-Gascón, Rom-Rodríguez y Santana-López, 2016), centrado en el modelo objetivo de métrica (Fondevila-Gascón, 2014a).

Son escasas las experiencias comerciales, a título de prueba o mediante emisión real, que se han llevado a cabo utilizando el nuevo estándar. Las reticencias de las agencias de medios y los controladores de audiencias, renuentes a modificaciones en el actual status quo audiovisual, limitan el alcance de las experiencias. Además, para llevar a cabo una implementación de publicidad interactiva en una cadena, hay que armonizar al actor creativo (el creador del anuncio), la empresa controladora de los datos digitales y la métrica, la agencia de medios, la empresa anunciante y la cadena de televisión. Esa cadena es difícil de orquestar, y de ahí el mérito de investigaciones que permitan materializarla y que, a la vez, alumbren resultados reales extrapolables a una hipotética emisión real.

\section{Marco teórico}

La evolución del HbbTV se produce en el apartado cuantitativo, gracias a la mayor penetración del parque de televisores que disponen de ese estándar, y en el cualitativo, a raíz de la evolución de las opciones interactivas congénitas a la tecnología. La expansión de equipos con HbbTV es acusada, ya que dispone de 18 millones de televisores conectados y penetración intensa en mercados propensos en los últimos años, como Alemania (Welker, 2015), Austria, España, Estonia, Francia (Tobin, 2013), Holanda, Polonia y Suiza (CECABLE, 2017). En el caso alemán, el $84 \%$ de las pantallas vendidas con un tamaño superior a 50 pulgadas disponían de HbbTV. En cambio, para televisores de 32 pulgadas, la cifra se reducía al 24\%. En 2015, en España se vendieron 1,06 millones de televisores HbbTV, de forma que a inicios de 2017 el parque era de 4,5 millones de hogares con televisión conectada gracias al estándar HbbTV. El 85\% de ellos se conectan mediante HbbTV 1.2.1.

Las ventas internacionales se situaban en 231,3 millones de unidades en 2017 (GFK, 2017). La tendencia es a grandes formatos de pantalla, por encima de la 60 pulgadas. Para 2017 se preveía un 6\% de ventas de este tipo y un 35\% de televisores con resolución $4 \mathrm{~K}$ UHD. La televisión conectada acostumbra a ser antónimo de conexión a Internet. No obstante, un 47\% de ventas en 2017 corresponderán a la tipología smart.

Cualitativamente, la versión del estándar HbbTV 2.0.1, publicada en 2016, aporta un canal de retorno entre los receptores y el emisor. De hecho, ya se registraban desarrollos en Italia y el Reino Unido. El 2.0.1, que debía compatibilizarse con los anteriores, dota de sincronización, multidispositivo y HTML5 a la televisión interactiva, y genera más aplicaciones para el usuario.

De hecho, en el uso de las smart tv, el 53\% de los alemanes conectan el televisor a Internet. En el caso español, la cifra es del 48\%. Los servicios más utilizados son aplicaciones de catch up (vídeos bajo demanda con contenidos disponibles durante unos días posteriores a la emisión, como en Alacarta de RTVE), el propio Video on Demand (VoD), YouTube, y accesos a Netflix y Amazon. En el caso español, en los Juegos Olímpicos de Río 2016 los espectadores podían acceder a 19 señales en directo mediante streaming (DASH + HLS). Ello arrojó un balance de banda ancha de 350 TB en vivo y 55 TB en vídeo bajo demanda.

Otros servicios de comunicación innovadores en España que necesitan el flujo de banda ancha son el de Radio Nacional de España para distribuir programas radiofónicos con sonido Dolby Digital (DD+), el servicio de contenidos en $4 \mathrm{~K}$, la propuesta de EiTB (Euskadi) en HbbTV para cubrir las elecciones autonómicas de 2016 y el de TV3 (Cataluña) para subtitular a partir de EBU-TT. La noruega NRK utiliza el estándar HbbTV 2.0.1 para accesibilidad, e investiga sobre un protocolo dual que permita a las aplicaciones comunicarse entre ellas y con otros dispositivos.

Como el espectador desea acceder al contenido elegido en cualquier lugar y momento, la programación clásica de contenido cederá protagonismo. La demanda de información y de contenidos brotados de Internet refuerza la idea de la hibridación, de la suma de Internet y televisión. El HbbTV es utilizado para acceder a contenido a la carta por parte del 58\% de clientes de HbbTV; el 57\% ven vídeos en YouTube y el $48 \%$ visualizan otras plataformas de vídeo bajo demanda (GFK, 2017).

HbbTV Association y Smart TV Alliance estaban cooperando para ampliar la especificación HbbTV hacia los servicios Over-The-Top (OTT). El HbbTV permite alumbrar señales en streaming y enseñarlas en el televisor. Ello flexibiliza la opción de cubrir contenido de forma simultánea desde diferentes localizaciones. También refuerza el triple play (Fondevila-Gascón, 2009a), el quadruple play y el quintuple play (televisión, telefonía fija y móvil, Internet fijo y móvil), incluso en operadores de cable histórico (Fondevila-Gascón, 2004, 2009b).

El HbbTV (fundado por ANT Software Limited, EBU, France Télévisions, Institut für Rundfunktechnik GmbH, OpenTV Inc, Koninklijke Philips Electronics N.V Inc., Samsung, SES ASTRA 
S.A, Sony Corporation y TF1) concentra e empresas de radiodifusión y de normalización, editoras de software middleware para dispositivos de electrónica de consumo y fabricantes y laboratorios de prueba para dispositivos de electrónica de consumo. Las pruebas de publicidad interactiva en HbbTV se inician en 2014 y 2015, en los canales alemanes ProSieben y RTL.

Las métricas que facilita el HbbTV adquieren más relevancia gracias al control en tiempo real y al detector sin watermarking, técnica de ocultación de datos que delata posibles usos ilícitos de servicios digitales. Una métrica esencial es el Click Through Rate (CTR), la división entre clics e impresiones, es decir, entre las veces que un anuncio aparece en el campo visual del telespectador y las que entra en la propuesta. Al tratarse de interactividad, la postura del consumidor es más activa que en el caso de anuncios convencionales. Las cifras habituales de CTR se sitúan en una horquilla entre el 0,1\% y 0,3\% en Internet y entre el 0,08\% y 0,1\% en televisión (GTO Telecom, 2016). Por ende, se trata de unos resultados muy modestos, que deben impulsar a las agencias publicitarias y de medios a buscar fórmulas alternativas más rentables. En esa encrucijada aparecen las campañas de publicidad interactiva en HbbTV, que en algunos casos han alcanzado cifras por encima del $6 \%$.

Otro indicador necesario para calcular el CTR es la cantidad de impresiones totales de campaña (viewability). Igualmente es preciso conocer el número de ocasiones en las que se clican los botones para acceder al contenido publicitario y al de aviso legal.

La frecuencia de accesos a HbbTV, esto es, la cantidad de impactos por dispositivo único, aporta valor en términos de frecuencia. Para según qué tipología de producto anunciado (sobre todo si es tecnológico o de uso complejo), se necesitará un número de impactos mayor que para un producto sencillo o commodity. En este caso se antepondrá el alcance a la frecuencia, en términos de Gross Rating Point (GRP).

También adquiere relevancia el tiempo promedio de reproducción del vídeo, una vez se ha conseguido captar la atención del telespectador. En la industria comunicativa, tanto en la periodística como en la publicitaria, el indicador de referencia centrado en el clic (el Coste por Clic, por ejemplo) está dejando paso al del tiempo de permanencia, sea el Cost per Hour (CPH), sea el Committed Visitor Volume (CVV). Se prioriza un contacto prolongado con el cliente, ya que ello redunda en más opciones de seducción y venta y, a la par, en más oportunidades de fidelización. Un telespectador fidelizado puede convertirse, con facilidad, en un evangelizador de la marca anunciada en todos los ámbitos, comenzando por las redes sociales.

El carácter reciente de la implantación del estándar HbbTV (Fondevila-Gascón, 2012a) explica que las investigaciones alusivas sean escasas, centradas en aplicaciones educativas (Fondevila-Gascón et al., 2017b), en el servicio de TV a la carta (Mansilla y Marcos-Mora, 2013), en la navegación geoespacial para vídeos en función de su ubicación geográfica según el etiquetado (Berger et altri, 2014), en las televisivas en el caso alemán (Botey et al., 2016) y en aplicaciones publicitarias (Fondevila-Gascón et al., 2017a; 2017b; Fondevila-Gascón, Botey-López, Rom-Rodríguez, y Muñoz-González, 2017a; 2017b) y comparativas publicitarias interactivas (Fondevila-Gascón, Botey-López y Rom-Rodríguez, 2017a; 2017b).

\section{Metodología}

El objetivo general de la investigación es analizar el impacto del HbbTV en campañas publicitarias interactivas. Las hipótesis a validar o refutar son las siguientes:

H1. El telespectador tiende a clicar el botón de aviso legal (el botón verde).

H2. Existe una relación entre el tiempo promedio de reproducción del vídeo en segundos y la voluntad de querer participar.

H3. Existe un equilibrio entre las diferentes campañas a la hora de impulsar el clic del telespectador.

La metodología de la investigación es cuantitativa y cualitativa, es decir, que se opta por la triangulación. Así, desde el punto de vista cuantitativo, la empresa GCO Telecom colaboró con el Grupo de Investigación GRECC de la Universitat Ramon Llull, el Grupo de Investigación sobre Periodismo y Marketing Digital y Banda Ancha y el Grupo de Investigación sobre Sistema Innovadores de Monetización de Periodismo, Marketing y Turismo Digital (SIMPED), de la Universitat de Girona y el Centre d'Estudis sobre el Cable (CECABLE), para llevar a cabo una prueba real.

Las campañas planteadas son cuatro propuestas diferenciadas en los paneles de medida de CTR. Se suministró a la cadena de televisión una muestra con cuatro anuncios de un producto de belleza de Henkel.

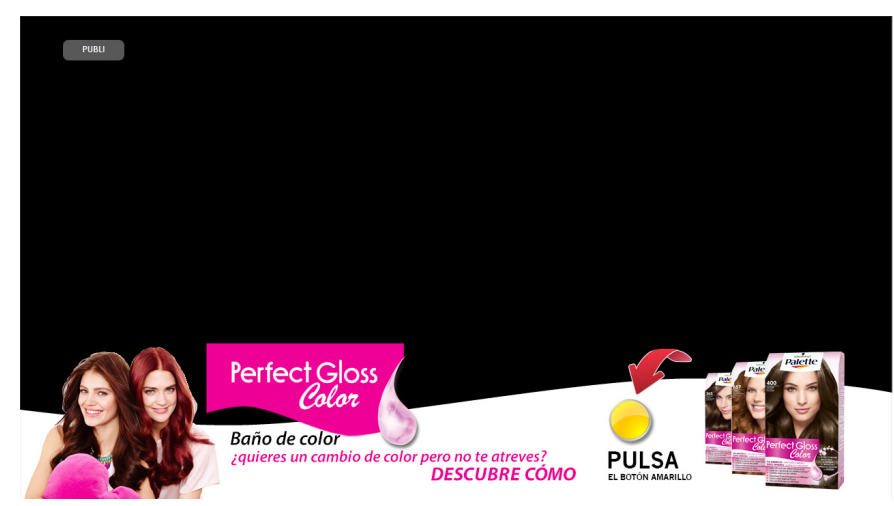

Figura 1. Color 1 (faldón baño de color con grafismo completo). Elaboración de Blanquerna-URL. 


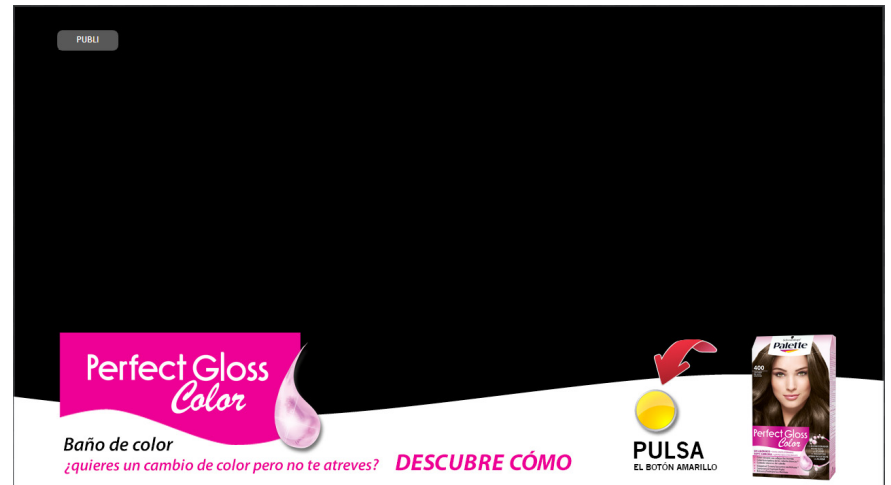

Figura 2. Color 2 (faldón baño de color minimalista). Elaboración de Blanquerna-URL.

El anuncio A (Figura 1) era un faldón (en la zona inferior de la pantalla) en el que aparecen tres chicas y en el que se anuncia el baño de color.

El anuncio B (Figura 2) era un faldón (en la zona inferior de la pantalla), sin chicas y en el que se anuncia el baño de color.

El anuncio C (Figura 3) era un faldón (en la zona inferior de la pantalla), en el que aparecen tres chicas y en el que se anuncia un tono sobre tono.

El anuncio D (Figura 4) era un faldón (en la zona inferior de la pantalla) sin chicas y en el que se anuncia el tono sobre tono.

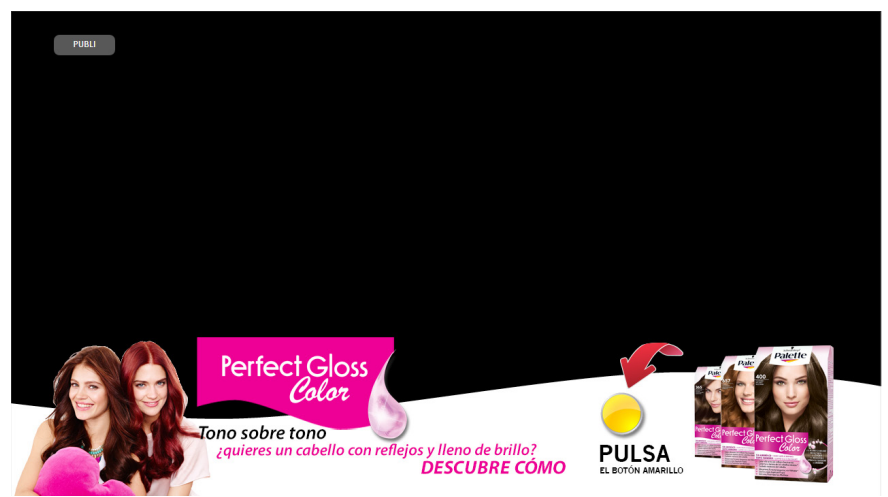

Figura 3. Tono 1 (faldón tono sobre tono con grafismo completo). Elaboración de Blanquerna-URL.

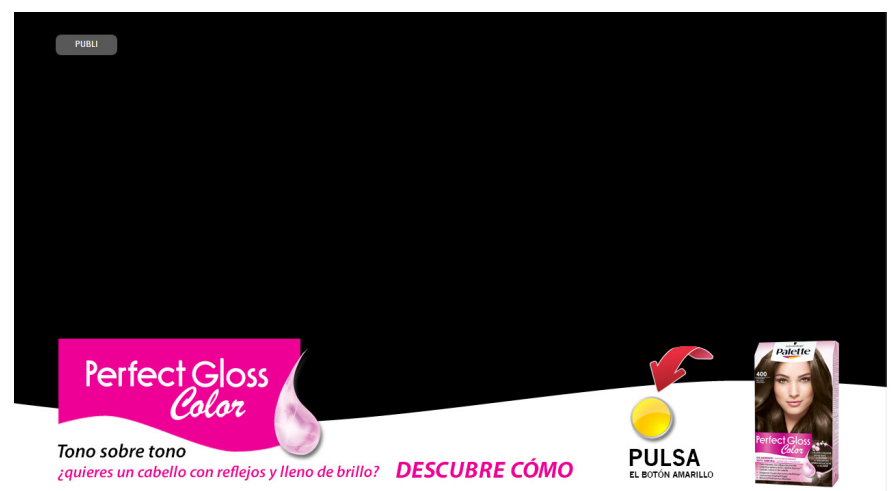

Figura 4. Tono 2 (faldón tono sobre tono minimalista). Elaboración de Blanquerna-URL.
Para cada campaña se mide lo siguiente: CTR (Click Through Rate); número de impresiones totales de campaña; número de veces que se aprieta el botón de aviso legal; tiempo promedio de reproducción del vídeo.

La duración del faldón en pantalla es de 30 segundos. En cada televisor se configuró un frequency capping de 12 en el caso de que no se haya llegado a ver todo el vídeo. Si lo ve íntegro en algún impacto ya no se impacta más a ese espectador. El frequency capping es la limitación de frecuencia, término en publicidad que significa la restricción o limitación en el número de veces (frecuencia) en el que a un visitante a un sitio web específico o a una campaña se le muestra un anuncio en particular. Esta restricción se aplica a todos los sitios web o pantallas HbbTV que ofrecen anuncios de la misma red de publicidad. La limitación de frecuencia es una característica dentro de la publicación de anuncios que permite limitar el número máximo de impresiones que un visitante puede ver un anuncio específico dentro de un período de tiempo. Así, 5 views / visitante / 24 horas significa que, después de ver este anuncio 5 veces, ese visitante no lo verá de nuevo durante 24 horas. Esta función utiliza cookies para recordar el recuento de impresión. En nuestro experimento, el tiempo entre impacto a un mismo televisor es de 3 horas.

El scheduler que dispara la publicidad sobre KissTV está programado para lanzar eventos cada 5 minutos a todos los aparatos de televisión conectados durante todo el día.

En las tablas se observan diversas variables. La primera es la ID de campaña (Campaign ID), que es el identificador de cada uno de los cuatro anuncios. La columna de Watched Time recoge el tiempo de visión del anuncio, en segundos. El televidente, una vez visionado el faldón, podía clicar en el botón amarillo y ver el anuncio. Como los anuncios ocupaban 115 segundos, esa es la máxima duración del seguimiento.

La columna de Last Event Type presenta la opción de mostrar el faldón y de clicar el botón amarillo. La variable 8 es el visionado del faldón; la 9, clicar el botón amarillo; la 10, ver el vídeo (más allá de los segundos de visionado); la 11, los segundos de visionado del vídeo; y la 12, el clic al botón verde (aviso legal).

En la columna Button Yellow Clicks, la opción es binaria: o se clica (1) o no se clica (0). Se trata del botón que permite visionar el anuncio. En el caso de Green Yellow Clicks, se repite la fórmula binaria. El botón verde permite leer el aviso legal de la campaña. Por tanto, no son acumulativos, sino que se puede clicar el botón amarillo sin clicar el verde, el verde sin clicar el amarillo, se pueden clicar ambos botones o ninguno.

\section{Resultados}

En la investigación sobre publicidad interactiva en televisión se llevaron a cabo 4 campañas (CampaignID), que se diferenciaron en los paneles de medida de CTR (Tabla 1), según se 
tratase de faldón baño de color grafismo completo, faldón baño de color minimalista, faldón baño sobre tono grafismo completo o faldón baño sobre tono minimalista.

\begin{tabular}{|l|l|}
\hline Campaña & Porcentaje \\
\hline $\begin{array}{l}\text { 28-C1 Faldón baño de color grafismo } \\
\text { completo }\end{array}$ & $32 \%$ \\
\hline 29-C2 Faldón baño de color minimalista & $25,4 \%$ \\
\hline $\begin{array}{l}\text { 30-T1 Faldón baño sobre tono grafismo } \\
\text { completo }\end{array}$ & $21,3 \%$ \\
\hline 31-T2 Faldón baño sobre tono minimalista & $21,3 \%$ \\
\hline
\end{tabular}

Tabla 1. Paneles de medida de las campañas propuestas. Fuente: Elaboración propia.

Se detectó que la reacción de los telespectadores a cada anuncio cambiaba. No obstante, la mayor parte de los espectadores no reaccionaron a las campañas en la primera muestra de los faldones (Tabla 2).

\begin{tabular}{|l|l|}
\hline Reacción & Porcentaje \\
\hline 8-Ve la campaña pero no reacciona & $97,8 \%$ \\
\hline 9-Aprieta el botón amarillo una o más veces & $0,3 \%$ \\
\hline $\begin{array}{l}\text { 10-Se dispara el vídeo, pero no envía resul- } \\
\text { tado, habiendo pulsado el botón amarillo o no }\end{array}$ & $0,1 \%$ \\
\hline $\begin{array}{l}\text { 11-Pulsa OK, pero no hace o escribe nada. } \\
\text { Puede haber visto el vídeo }\end{array}$ & $1,4 \%$ \\
\hline $\begin{array}{l}\text { 12-Ve el aviso legal. Normalmente ha visto } \\
\text { segundos del vídeo }\end{array}$ & $0,4 \%$ \\
\hline
\end{tabular}

Tabla 2. Respuesta del televidente a la primera muestra de la campaña. Fuente: Elaboración propia.

Se obtuvo un tiempo promedio de reproducción del vídeo (watchedTime) de 35.31 segundos ( $D E=41.55)$. La oscilación en segundos abarca de 1 a 158 segundos (en 583 casos). No se dio reproducción en 24.768 casos de un total de 25.351 registros, es decir, en el $97,70 \%$ de los casos. Por tanto, la mayor parte de telespectadores no reprodujeron el anuncio, resultado que se alinea con las métricas digitales en su fase inicial.

En cuanto al rango de impresiones totales de campaña (Show Campaign_Impressions), oscila entre 1 y 37 veces. La cantidad de ocasiones en que se pulsa el botón de aviso legal, esto es, el botón verde, fluctúa entre 0 y 9 veces. La pulsión del botón de participación, es decir, el amarillo, oscila entre 0 y 3 veces. Como la proporción de individuos que clicaron repetidamente el botón amarillo (0,1\%) o verde (0,03\%) es insignificante, se recalcularon en variables binarias $(0 / 1=$ no/sí clic; BYC el amarillo, BGC para el verde).

Con relación al impacto de la campaña, se observan algunas variaciones, no substanciales, según la campaña propuesta. El tipo de evento condiciona las variables de impacto (Tablas 3 y 4) y se asocia con la variable promedio de tiempo de visionado del vídeo en segundos ( $F=164.924, p<0.0001)$. El tiempo promedio es mayor en el grupo último evento: el telespectador clica el botón verde y lee aviso legal (número 12). De esta forma, se confirma H1 (El telespectador tiende a clicar el botón de aviso legal).

El clic sobre botón verde ( $F=312.89, p<0.0001)$ es más probable cuando se dispara el vídeo, pero no se envía resultado, se haya pulsado o no el botón amarillo (grupo 10), cuando se pulsa OK (pero no hace o escribe nada), con lo que se puede haber visto el vídeo (grupo 11), cuando se ve el aviso legal y normalmente han visionado segundos del vídeo (grupo 12).

El clic en el botón amarillo ( $F=10222.43, p<0.0001)$ es más probable en todos los grupos excepto en el 8 . Así, el 0,8\% en el tipo de evento ven la campaña pero no reaccionan (no suelen hacer nada ante el anuncio). La probabilidad es del 100\% en el 9, puesto que es el grupo que da OK al botón amarillo.

\begin{tabular}{|l|c|c|c|c|}
\hline \multicolumn{2}{|c|}{ Tipo campaña } & $n$ & Media & $\begin{array}{c}\text { Desviación } \\
\text { estándar }\end{array}$ \\
\hline \multirow{4}{*}{$\begin{array}{l}\text { Watched } \\
\text { Time }\end{array}$} & 28 & 155 & 38,56 & 44,622 \\
\cline { 2 - 5 } & 29 & 160 & 32,17 & 36,846 \\
\cline { 2 - 5 } & 30 & 122 & 33,33 & 40,925 \\
\cline { 2 - 5 } & 31 & 146 & 36,97 & 43,579 \\
\hline \multirow{4}{*}{ BYC } & Total & 583 & 35,31 & 41,547 \\
\cline { 2 - 5 } & 28 & 8050 &, 0243 &, 15414 \\
\cline { 2 - 5 } & 29 & 6446 &, 0264 &, 16025 \\
\cline { 2 - 5 } & 30 & 5400 &, 0254 &, 15726 \\
\hline \multirow{4}{*}{ BGC } & 31 & 5455 &, 0321 &, 17623 \\
\cline { 2 - 5 } & 28 & 8050 &, 0011 &, 03342 \\
\cline { 2 - 5 } & 29 & 6446 &, 0008 &, 02784 \\
\cline { 2 - 5 } & 30 & 5400 &, 0013 &, 03598 \\
\cline { 2 - 5 } & 31 & 5455 &, 0018 &, 04278 \\
\hline & Total & 25351 &, 0012 &, 03495 \\
\hline
\end{tabular}

Tabla 3. Descripción de variables de impacto de la campaña. Fuente: Elaboración propia.

El retorno de las campañas en tiempo promedio de reproducción del vídeo en segundos no cambia según el anuncio ( $F=$ 0.790, $p=0.5$ ), así como la probabilidad de ver el aviso legal ( $F$ $=0.938, p=0.421)$. En contraposición, se observa vínculo entre el tiempo promedio de reproducción del vídeo en segundos y la predisposición a la participación (Tablas 5 y 6) sobre el botón amarillo ( $F=2.724, p=0.043$ ). Ello corrobora H2 (Existe una relación entre el tiempo promedio de reproducción del vídeo en segundos y la voluntad de querer participar).

En los contrastes aplicados (tipo Bonferroni) se observan diferencias significativas sólo entre los grupos o campañas 28 (Faldón baño de color grafismo completo) respecto a la 31 (T2 


\begin{tabular}{|c|c|c|c|c|c|c|}
\hline \multirow{2}{*}{\multicolumn{2}{|c|}{ Tipo del Último evento }} & \multirow{3}{*}{$\begin{array}{c}n \\
147 \\
\end{array}$} & \multirow{3}{*}{$\begin{array}{l}\text { Media } \\
23,22 \\
\end{array}$} & \multirow{3}{*}{$\begin{array}{c}\begin{array}{c}\text { Desviación } \\
\text { estándar }\end{array} \\
\text { Límite inferior } \\
29,313 \\
\end{array}$} & \multicolumn{2}{|c|}{$\begin{array}{l}95 \% \text { del intervalo de con- } \\
\text { fianza para la media }\end{array}$} \\
\hline & & & & & Límite superior & Límite superior \\
\hline \multirow{6}{*}{ WatchedTime } & 8 & & & & 18,44 & 28,00 \\
\hline & 9 & 2 & 8,50 & ,707 & 2,15 & 14,85 \\
\hline & 10 & 15 & 23,00 & 33,562 & 4,41 & 41,59 \\
\hline & 11 & 332 & 22,43 & 27,453 & 19,47 & 25,39 \\
\hline & 12 & 87 & 107,64 & 30,209 & 101,21 & 114,08 \\
\hline & Total & 583 & 35,31 & 41,547 & 31,93 & 38,69 \\
\hline \multirow{6}{*}{ BYC } & 8 & 24823 & ,0083 & ,09072 & ,0072 & ,0094 \\
\hline & 9 & 71 & 1,0000 & .00000 & 1,0000 & 1,0000 \\
\hline & 10 & 15 & 8000 & .41404 & .5707 & 1,0293 \\
\hline & 11 & 353 & ,8584 & ,34918 & 8218, & ,8949, \\
\hline & 12 & 89 & ,9663 & 18150, & ,9281 & 1,0045 \\
\hline & Total & 25351 & ,0267 & ,16134 & ,0248 & ,0287 \\
\hline \multirow{6}{*}{ BGC } & 8 & 24823 &, 0002 & ,01419 & ,0000 & ,0004 \\
\hline & 9 & 71 & ,0000 & 00000 & ,0000 & , 0000 \\
\hline & 10 & 15 & ,0667 & ,25820 &,- 0763 & 2097 \\
\hline & 11 & 353 & ,0538 & 22599, & ,0302 & ,0775 \\
\hline & 12 & 89 &, 0674 & ,25216 &, 0143 &, 1205 \\
\hline & Total & 25351 & ,0012 & ,03495 & ,0008 & ,0017, \\
\hline
\end{tabular}

Tabla 4. Descripción de variables de impacto de la campaña, dentro de los tipos de último evento. Fuente: Elaboración propia.

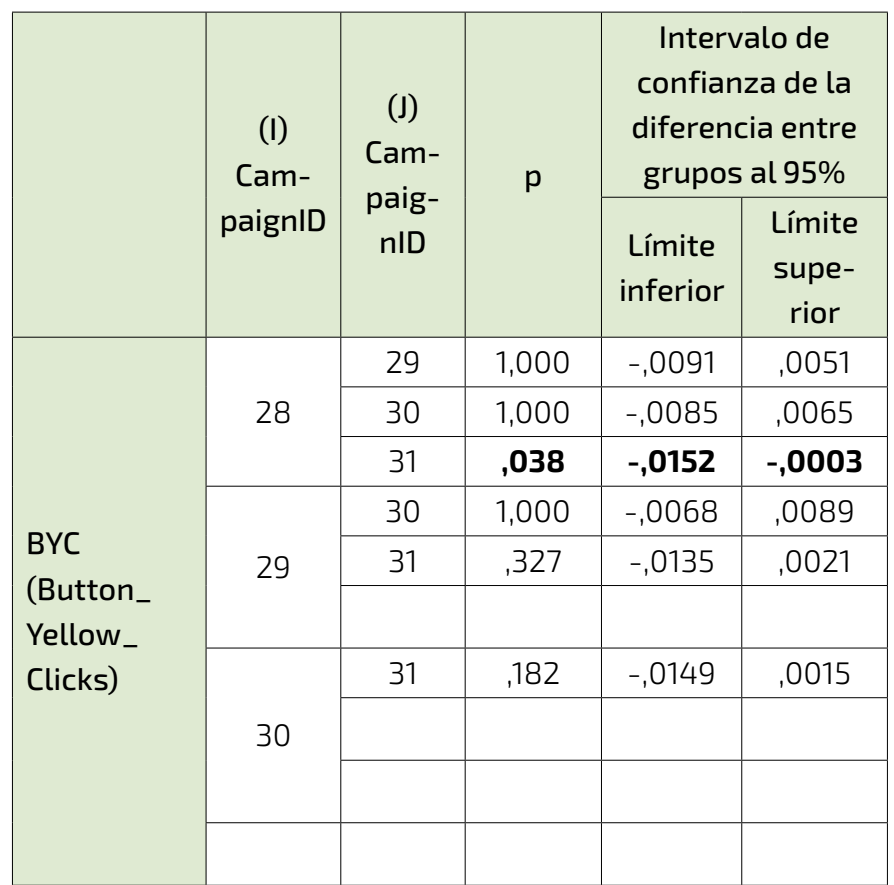

Tabla 5. Contrastes tipo Bonferroni para BYC (Button_Yellow_ Clicks) entre campañas. Fuente: Elaboración propia.

Faldón baño sobre tono minimalista). La campaña 31, la más modesta gráficamente, se reveló más efectiva en probabilidad de participar o clicar el botón amarillo (IC = 0.0152 a 0.0003). La probabilidad de participar de la campaña 31 supera a la 28. No se detectan diferencias significativas con relación al resto de campañas. Por eso se valida parcialmente H3 (Existe un equilibrio entre las diferentes campañas a la hora de impulsar el clic del telespectador), aunque la propuesta sencilla (la de la campaña 31) consigue más impacto que la compleja (la de la campaña 28).

Por ende, los anuncios donde aparecen modelos (presencia humana) no tienden más al clic, con las reservas propias del grafismo que se utilice. Así, en otro tipo de ejemplo el balance puede ser distinto. Se prioriza en este caso el equilibrio dentro del formato faldón, lo que lleva a pensar que, de cara a las empresas anunciantes, habría que buscar mayor probabilidad de clic en otro tipo de formatos, no en variaciones dentro del mismo formato.

\section{Conclusiones}

De esta investigación se desprende que el receptor que quiere participar en actividades interactivas clica el botón amarillo antes de la tercera oportunidad. En este caso, un 95\% clican entre la segunda y la tercera opciones. Tras ello, los intentos de atrapar al telespectador no cuajarán, por lo que invertir recursos puede implicar un efecto bumerán.

En general, el consumidor que clica el botón de aviso legal, es decir, el botón verde, clica también el de participación, el amarillo. La creciente sensibilización en la Unión Europea por la protección de datos y los derechos de autor digitales explica 


\begin{tabular}{|c|c|c|c|c|c|c|c|}
\hline & & & Dif. & $E E$ & $p$ & $\begin{array}{l}\text { Límite } \\
\text { inferior }\end{array}$ & $\begin{array}{l}\text { Límite } \\
\text { superior }\end{array}$ \\
\hline \multirow{16}{*}{ WatchedTime } & \multirow{4}{*}{8} & 9 & 14,718 & 20,282 & 1,000 & $-42,43$ & 71,87 \\
\hline & & 10 & ,218 & 7,722 & 1,000 & $-21,54$ & 21,98 \\
\hline & & 11 & ,787 & 2,823 & 1,000 & $-7,17$ & 8,74 \\
\hline & & 12 & $-84,426^{*}$ & 3,854 & ,000 & $-95,29$ & $-73,57$ \\
\hline & \multirow{4}{*}{9} & 8 & $-14,718$ & 20,282 & 1,000 & $-71,87$ & 42,43 \\
\hline & & 10 & $-14,500$ & 21,447 & 1,000 & $-74,93$ & 45,93 \\
\hline & & 11 & $-13,931$ & 20,206 & 1,000 & $-70,87$ & 43,01 \\
\hline & & 12 & $-99,144^{*}$ & 20,376 & ,000 & $-156,56$ & $-41,73$ \\
\hline & \multirow{4}{*}{10} & 8 &,- 218 & 7,722 & 1,000 & $-21,98$ & 21,54 \\
\hline & & 9 & 14,500 & 21,447 & 1,000 & $-45,93$ & 74,93 \\
\hline & & 11 & .569 & 7,521 & 1,000 & $-20,62$ & 21,76 \\
\hline & & 12 & $-84,644^{*}$ & 7,965 & ,000 & $-107,09$ & $-62,20$ \\
\hline & \multirow{4}{*}{11} & 8 &,- 787 & 2,823 & 1,000 & $-8,74$ & 7,17 \\
\hline & & 9 & 13,931 & 20,206 & 1,000 & $-43,01$ & 70,87 \\
\hline & & 10 &,- 569 & 7,521 & 1,000 & $-21,76$ & 20,62 \\
\hline & & 12 & $-85,213^{*}$ & 3,431 &, 000 & $-94,88$ & $-75,54$ \\
\hline \multirow{16}{*}{ BYC } & \multirow{4}{*}{8} & 9 &,$- 99170^{*}$ & ,01186 &, 000 & $-1,0250$ &,- 9584 \\
\hline & & 10 &,$- 79170^{*}$ &, 02578 &, 000 &,- 8641 &,- 7193 \\
\hline & & 11 &,$- 85006^{\star}$ &, 00535 & ,000 &,- 8651 &,- 8350 \\
\hline & & 12 &,$- 95799^{*}$ & 01060 & ,000 &,- 9877 &,- 9282 \\
\hline & \multirow{4}{*}{9} & 8 & ,99170* & ,01186 & ,000 & ,9584 & 1,0250 \\
\hline & & 10 & $20000^{*}$ &, 02836 & ,000 & ,1204 & 2796 \\
\hline & & 11 &, $14164^{*}$ & ,01298 & ,000 & ,1052 & 1781 \\
\hline & & 12 & 03371 & ,01588 &, 338 &,- 0109 & ,0783 \\
\hline & \multirow{4}{*}{10} & 8 &, $79170^{*}$ & ,02578 & ,000 & ,7193 & ,8641 \\
\hline & & 9 &,$- 20000^{*}$ &, 02836 & ,000 &,- 2796 &,- 1204 \\
\hline & & 11 &,- 05836 &, 02631 &, 266 &,- 1322 & ,0155 \\
\hline & & 12 &,$- 16629^{*}$ & ,02786 &, 000 &,- 2445 &,- 0881 \\
\hline & \multirow{8}{*}{11} & 8 &, $85006^{*}$ &, 00535 & ,000 & ,8350 & ,8651 \\
\hline & & 9 &,$- 14164^{*}$ & ,01298 & ,000 &,- 1781 &,- 1052 \\
\hline & & 10 & ,05836 & ,02631 &, 266 &,- 0155 & ,1322 \\
\hline & & 12 &,$- 10794^{*}$ & ,01184 &, 000 &,- 1412 &,- 0747 \\
\hline \multirow{16}{*}{ BGC } & & 9 & ,00020 &, 00405 & 1,000 &,- 0112 &, 0116 \\
\hline & & 10 &,$- 06647^{*}$ & ,00881 & ,000 &,- 0912 &,- 0417 \\
\hline & & 11 &,$- 05362^{*}$ & ,00183 & ,000 &,- 0588 &,- 0485 \\
\hline & & 12 &,$- 06721^{*}$ &, 00362 & ,000 &,- 0774 &,- 0570 \\
\hline & \multirow{4}{*}{9} & 8 &,- 00020 &, 00405 & 1,000 &,- 0116 & 0112 \\
\hline & & 10 &,$- 06667^{*}$ & ,00970 & ,000 &,- 0939 &,- 0394 \\
\hline & & 11 &,$- 05382^{*}$ &, 00444 & ,000 &,- 0663 &,- 0414 \\
\hline & & 12 &,$- 06742^{*}$ &, 00543 & ,000 &,- 0827 &,- 0522 \\
\hline & \multirow{4}{*}{10} & 8 &, $06647^{*}$ & ,00881 & ,000 & 0417 & 0912 \\
\hline & & 9 &, $06667^{*}$ & ,00970 &, 000 & ,0394 & 0939 \\
\hline & & 11 & ,01284 & ,00899 & 1,000 &,- 0124 & ,0381 \\
\hline & & 12 &,- 00075 & ,00952 & 1,000 &,- 0275 &, 0260 \\
\hline & \multirow{4}{*}{11} & 8 &, $05362^{*}$ & ,00183 & ,000 & ,0485 & ,0588 \\
\hline & & 9 &, $05382^{\star}$ &, 00444 & ,000 & 0414 & ,0663 \\
\hline & & 10 &,- 01284 & ,00899 & 1,000 &,- 0381 &, 0124 \\
\hline & & 12 &,$- 01359^{*}$ & ,00405 & ,008 &,- 0250 &,- 0022 \\
\hline
\end{tabular}

Tabla 6. Contrastes tipo Bonferroni para WatchedTime, BYC y BGC entre tipos de último evento. Fuente: Elaboración propia. 
esos resultados. Igualmente, la costumbre de consultar documentos legales, habitual en actividades digitales como el comercio electrónico, se retroalimenta con otras modalidades virtuales.

También se observa una relación entre el tiempo promedio de reproducción del vídeo en segundos y la voluntad de querer participar. Los más avezados o llamados a la acción son más propensos al anuncio, que, no obstante, rara vez se consume hasta su conclusión. La predisposición a la participación conlleva más probabilidad de consumir segundos de anuncio. El Committed Visitor Volum (CVV), pues, se asocia a esa voluntad participativa.

Contra lo que la intuición podría indicar, el faldón más sencillo es el que concita más interés entre la audiencia. Ello confirma el equilibrio entre las diversas campañas cuando se trata de impulsar el clic del telespectador. Al menos en esta investigación, el factor de creatividad no resulta decisivo a la hora de conseguir un retorno de la inversión, sea intangible o tangible.

Lógicamente, ese hallazgo requiere de nuevas pruebas cuantitativas, que podrían arrojar luz sobre el tipo de producto más susceptible de generar contrastes, el formato más proclive a ello (buscando diferencias significativas entre los formatos estáticos y los animados), o las franjas de edad más susceptibles de adentrarse en la interactividad.

\section{Referencias}

Abertis Telecom (2012). La televisión conectada. Una oportunidad para el sector audiovisual. Barcelona: Abertis.

Azcoitia, N. (2006). Diez años de publicidad interactiva. Harvard Deusto Márketing y Ventas, (73), 52-57.

Berger, A., Fritzsche, T., Heidt, M., y Eibl, M. (2014). Location Based Video Flipping: Navigating Geospatial. Videos in Lean Back Settings. TVX'14. Newcastle: UK. doi: 10.6084/m9.figshare.1032591

Botey-López, J., Fondevila-Gascón, J. F., Ordeix, E., y Rom-Rodríguez, J (2016). HBBTV y publicidad interactiva aplicada: el caso alemán. adComunica. Revista científica de estrategias, tendencias e innovación en comunicación, (12), 143-161. doi: 10.6035/21740992.2016 .12 .9

CECABLE (2017). Informe sobre penetración del HbbTV. Terrassa: CECABLE.

Del Ama-Gonzalo, J. C., Barceló-Ugarte, T., y Sánchez-Martínez, M. (2011). HbbTV hacia un modelo de convergencia en televisión. En M. Alcudia-Borreguero, J. M. Legorburu-Hortelano, L. López-Delgado, S. Ruíz-Gómez (Eds.), Competidores y aliados: medios de convergencia, los nuevos retos en comunicación (pp. 191-204). Madrid, España: CEU.

Fondevila-Gascón, J. F. (2004). Las redes de telecomunicaciones de cable histórico: realidad y tendencias. Revista de Comunicación de la SEECI (Sociedad Española de Estudios de la Comunicación Iberoamericana), 11(7), 67-89.

Fondevila-Gascón, J. F. (2009a). El peso de la televisión en el triple play de los operadores de cable en España y en Europa. ZER, Revista de Estudios de Comunicación, 14(27), 13-31.
Fondevila-Gascón, J. F. (2009b). La adaptación regulatoria de los operadores de cable histórico en España. La competencia de los grandes operadores. Telos. Cuadernos de Comunicación e Innovación, (80), 139-146.

Fondevila-Gascón, J. F. (2012a). Connected Television: Advantages and Disadvantages of the HbbTV Standard. Cuadernos de Información, (32), 11-20.

Fondevila-Gascón, J. F. (2012b). The Broadband Society in the World. Terrassa: CECABLE.

Fondevila-Gascón, J. F. (2014a). El uso de hipertexto, multimedia e interactividad en periodismo digital: propuesta metodológica de ranking de calidad. ZER, Revista de Estudios de Comunicación, 19(36), 55-76.

Fondevila-Gascón, J. F. (2014b). L'ús de la tecnologia als diaris de l'ACPC. Fórmules de millora de rendibilitat a través d'Internet (VII Premi de Recerca Associació Catalana de la Premsa Comarcal). Barcelona: Fundació Catalana de la Premsa Comarcal i Local.

Fondevila-Gascón, J. F. (2016). El HbbTV continúa avanzando. IPTV, (40), 22-24.

Fondevila-Gascón, J. F., Sierra-Sánchez, J., y Del Olmo-Arriaga, J. L. (2011). New communicative markets, new business models in the digital press. Trípodos, [Número extra], 301-310.

Fondevila-Gascón, J. F., Del Olmo-Arriaga, J. L., Beriain-Bañares, A., Carreras-Alcalde, M., y Pesqueira-Zamora, M. J. (2013). Educational possibilities in the Internet-television intersection: the HbbTV standard. International Conference on Education and New Developments 2013 (END 2013), Lisbon.

Fondevila-Gascón, J. F., Mir-Bernal, P., Carreras-Alcalde, M., y Seebach, S. (2015). HbbTV history and its educational possibilities: Teaching options in times of the Internet. En: M. Carmo y World Institute for Advanced Research and Science (WIARS) (Eds.), Education Applications \& Developments (pp. 103-112). Lisboa, Portugal: InScience Press, GIMA - Gestão de Imagem Empresarial.

Fondevila-Gascón, J. F., Rom-Rodríguez, J., y Santana López, E. (2016). Comparativa internacional del uso de recursos digitales en el periodismo digital deportivo: estudio de caso de España y Francia. Revista Latina de Comunicación Social, (71), 124-140.

Fondevila-Gascón, J. F., Botey-López, J., Sierra-Sánchez, J., Rom-Rodríguez, J., y Ordeix-Rigo, E. (2017a). Publicidad interactiva en HBBTV: estudio de caso. Madrid (VI Congreso Internacional de Investigadores Audiovisuales), Universidad Camilo José Cela.

Fondevila-Gascón, J. F., Botey-López, J., Rom-Rodríguez, J., OrdeixRigo, E., Muñoz-González, M., Berbel-Giménez, G., y Gutiérrez-Aragón, Ó. (2017b). Learning about interactivity and the mix internet and television: the case of HBBTV. INTED2017 (11th International Technology, Education and Development Conference), Valencia.

Fondevila-Gascón, J. F., Botey-López, J., Rom-Rodríguez, J., y MuñozGonzález, M. (2017a). Convergencia mediática y publicidad interactiva: aplicación práctica en HBBTV. II Congreso Internacional Comunicación y Pensamiento: Internet y redes sociales: nuevas libertades, nuevas esclavitudes. Grupo de Investigación en Estructura, Historia y Contenidos de la Comunicación (GREHCCO) y Laboratorio de Estudios en Comunicación (LADECOM) de la Facultad de Comunicación de la Universidad de Sevilla, Sevilla.

Fondevila-Gascón, J. F., Botey-López, J., Rom-Rodríguez, J., y MuñozGonzález, M. (2017b). "Posibilidades comunicativas del HbbTV: un nuevo escenario para la publicidad interactiva". En A. Castelló- 
Martínez y C. del Pino-Romero (Coords.), Publicidad y Convergencia Mediática. Nuevas estrategias de comunicación persuasiva (pp. 11-28). Sevilla: Egregius Ediciones.

Fondevila-Gascón, J. F., Botey-López, J., y Rom-Rodríguez, J. (2017a). Formats emergents en televisió: anàlisi comparativa d'aplicacions publicitàries interactives en HBBTV. Comunicació: Revista de Recerca i d'Anàlisi, 34(1), 67-81.

Fondevila-Gascón, J. F., Botey-López, J., y Rom-Rodríguez, J. (2017b). Campañas publicitarias interactivas en HBBTV: estudio de caso. Reality and screen: a postmodern mirror. IX International Conference on Communication and Reality, Barcelona (Universitat Ramon Llull).

GFK (2017). Informe sobre ventas de televisores HbbTV. Madrid: GFK.

GTO Telecom, 2016. Informe de datos CTR. Barcelona: GTO Telecom.

Highbeam Research (2018). Social Media Popularity Report. Chicago: HighBeam Research.

Mansilla, V., y Marcos-Mora, M. del C. (2013). Experiencia de usuario en televisión conectada: un estudio con usuarios. El profesional de la información, 22(2), 122-127.

Reding, V. (2007). The convergent Publisher-Print Media in the broadband economy. Brussels: Publishers Forum.

Reding, V. (2008). Europe on the way to a high speed Internet economy. Brussels: Launch Press EITO.

Tobin, A. (2013). Cloud over. Digital TV Europe, (312), 24-26.

Varona-Aramburu, D. (2014). El Botón Rojo de RTVE: una experiencia de implantación de HbbTV en España. Doxa Comunicación: revista interdisciplinar de estudios de comunicación y ciencias sociales, (19), 155-176.

Vázquez, J. L. (2013). La revolución de la televisión hibrida: la adopción del estándar HbbTV. Bit, 194.

Welker, K. (2015). Germany: ZAK takes fundamental decisions on platform regulation. IRIS: Legal Observations of the European Audiovisual Observatory, (9), 9-10.

\section{CV}

Joan Francesc Fondevila Gascón. Doctor en Periodismo por la Universidad Autónoma de Barcelona (2002) y Licenciado en Ciencias de la Información (rama Periodismo) y Licenciado en Ciencias Políticas y Sociología por la UAB. Es profesor titular de Universidad por Aneca y profesor agregado por AQU. Es profesor en la Universidad Pompeu Fabra, en la Facultad de Ciencias de la Comunicación y Relaciones Internacionales Blanquerna de la Universidad Ramon Llull, en la Escola Universitària Mediterrani (Universidad de Girona), en Centro Universitario Cesine, Universitat Oberta de Catalunya, Euncet, EAE Business School (Universidad Politécnica de Cataluña), Universidad de Barcelona y UAB. Imparte asignaturas de Grado y Postgrado sobre Periodismo y Comunicación Digital, Telecomunicaciones, Publicidad, Relaciones Públicas y Marketing, Logística y Operaciones y Comercio Electrónico. Sus líneas de investigación se centran en Periodismo y Marketing Digital y Telecomunicaciones y Banda Ancha. Es investigador principal del Grupo de Investigación sobre Periodismo y Marketing Digital y Banda Ancha y del Grupo de Investigación Sistemas
Innovadores de Monetización de Periodismo, Marketing y Turismo Digital. Ha publicado más de un centenar de artículos científicos indexados y numerosos libros y capítulos de libro, y ha recibido numerosos premios por su actividad de investigación, docente y de gestión. Es director del Centro de Estudios sobre el Cable (CECABLE), entidad que organiza las Jornadas del Cable y la Banda Ancha en Cataluña. Es autor del blog científico Telecomunicaciones y Periodismo, líder en sus áreas de conocimiento. http://www.telecomunicacionesyperiodismo. $\mathrm{com} /$

Jordi Botey López. Doctor en Publicidad y Relaciones Públicas por la Universidad Ramon Llull (2009) y Licenciado en Ciencias de la Comunicación rama Publicidad y Relaciones Públicas por la Universidad Autónoma de Barcelona. Estudios de Dirección de Marketing en la Escuela Superior de Dirección y Administración de Empresas, ESADE. Es profesor investigador adjunto de la Facultad de Ciencias de la Comunicación y Relaciones Internacionales Blanquerna de la Universidad Ramon Llull donde imparte asignaturas vinculadas al área de Planificación de Medios. Tutoriza Seminarios Especializados. Colabora, ocasionalmente, con otras Universidades y escuelas de negocios. Actualmente es Responsable de Relaciones con Empresas en el Vicedecanato de Empresa e Innovación en la FCRI Blanquerna. Ha trabajado más de 20 años como Director de Cuentas en agencias de publicidad como McCann Erickson, Bassat Ogilvy \& Mather, MMLB y Puzzle Newton. Adicionalmente y de modo puntual ejerce como consultor de comunicación para empresas y entidades públicas y privadas a través de su propia consultoría (Comunica.By Jordi Botey). Codirige un programa de radio en la emisora local Radio Ciutat Vella (Barcelona).

Josep Rom Rodríguez. Doctor en Publicidad y Relaciones Públicas por la Universidad Ramon Llull. Es vicedecano de la Facultad de Comunicación y Relaciones Internacionales y profesor en la Universitat Ramon Llull. Es investigador principal del Grupo de Investigación sobre Estrategia y Creatividad Publicitarias (GREC) y miembro del Grupo de Investigación sobre Periodismo y Marketing Digital y Banda Ancha y del Grupo de Investigación Sistemas Innovadores de Monetización de Periodismo, Marketing y Turismo Digital. Imparte docencia, desde el 1994, en el Grado de Publicidad y Relaciones Públicas, y en distintos másters y posgrados de la Facultad, así como en otros de la Universitat Pompeu Fabra (Máster U. Comunicación Gráfica), Universitat de Barcelona, Universidad de Sevilla (Dirección de Comunicación), Istituto Europeo di Design y Universidad Internacional Menéndez Pelayo. Es director del Máster Universitario en Estrategia y Creatividad Publicitarias de la Universidad Ramon Llull (desde 2008) y del Máster Universitario en Dirección de Arte en Publicidad. Especializado en el área de la Dirección de Arte en Publicidad, Creatividad, Comunicación institucional y Branding. 
Fátima Vila. Doctora en Comunicación por la Universidad Complutense de Madrid y licenciada en Periodismo por la Universidad de Sevilla, es Master en Marketing Online Internacional por la Escola Superior de Comerç - ESCI Pompeu Fabra y experta en Comunicación Corporativa y Propaganda Política por la Universidad de Sevilla. Es profesora en la EAE Business
School (Universidad Politécnica de Barcelona), Ostelea (Universitat de Lleida) y en Online Business School impartiendo y/o coordinando asignaturas de grado y másteres oficiales en Comunicación y Relaciones Públicas, Marketing y Comunicación Online y Turismo.

\section{MÁSTER UNIVERSITARIO ONLINE EN OCUMENTACIÓN DIGITAL} DISEÑA, CREA, GESTIONA, EVALÚA, COMPARTE Y PROMOCIONA RECURSOS DIGITALES

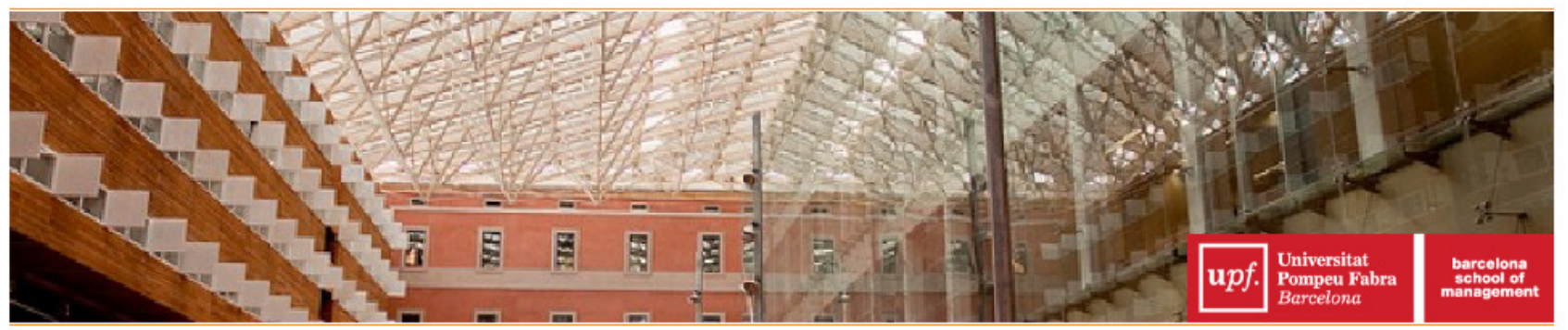

\section{MASTER EN USABILIDAD, DISEÑO DE INTERACCIÓN Y EXPERIENCIA DE USUARIO (ONLINE)}

MÁSTER EN UX

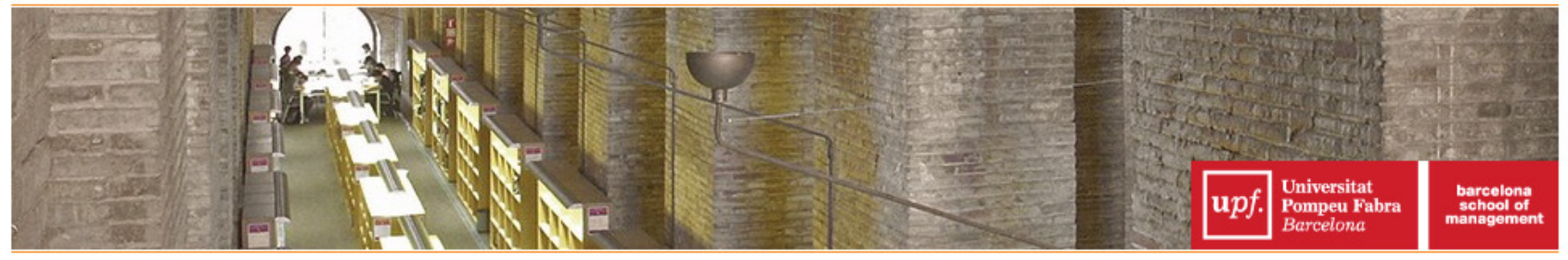

\section{MÁSTER EN BUSCADORES}

MARKETING (SEM) · POSICIONAMIENTO (SEO) . ANALYTICS . SOCIAL MEDIA

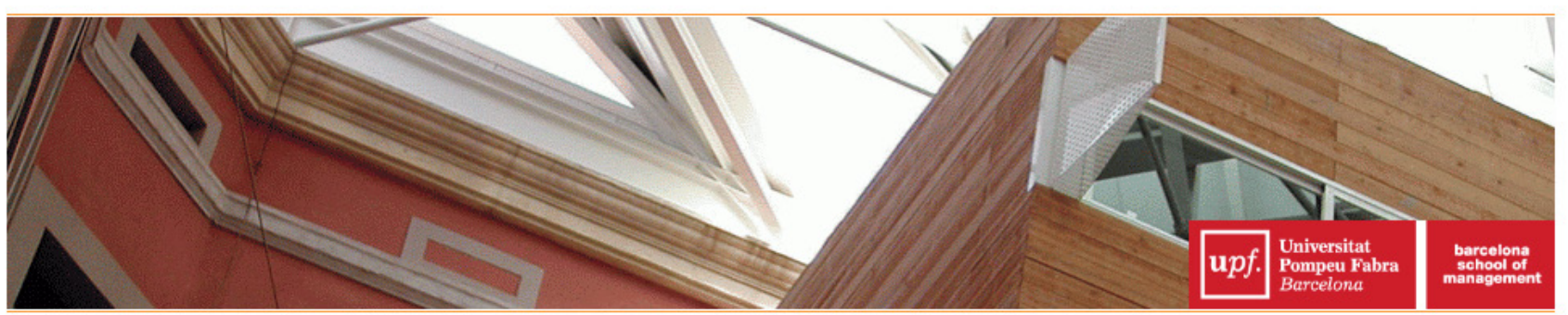

